\title{
Influence of Anticipation on Landing Patterns during Side-Cutting Maneuver in Female Collegiate Soccer Players
}

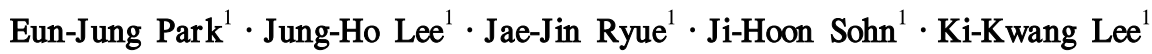 \\ ${ }^{1}$ Department of Physical Education, College of Physical Education, Kookmin University, Seoul, Korea
}

Received 31 July 2011; Received in revised form 12 August 2011; Accepted 12 December 2011

\begin{abstract}
To investigate the anticipatory effect on landing patterns during side-cutting maneuver, thirteen healthy female elite college soccer players participated in this study. Three-dimensional knee kinematics, effective mass and correlation between both these were measured and analyzed using a motion analysis and force plates. Each testing session included anticipated tasks, $45^{\circ}$ side-cutting tasks (AC), followed by a set of unexpected side-cutting (UC) in a random order. Knee flexion/extension, valgus/varus and internal/external rotation angles and effect mass were compared by using paired t-test. Also, correlation analysis was performed to identify the relationship between knee angles and effective mass. Effective mass during UC was greater than that during AC. Effective mass and maximum knee flexion angle were positively correlated during $\mathrm{AC}$ and not during UC. Based on the relationship between effective mass and knee flexion angle in $\mathrm{AC}$, shock absorption can be controlled by knee joint flexion in pre-predicted movement condition. However, effective mass can not be controlled by knee flexion in UC condition. The unexpected load affects were more irregular on the knee joint, which may be one of the injury mechanisms of anterior cruciate ligament (ACL) in female soccer players.
\end{abstract}

Keywords : Soccer, Anterior Cruciate Ligament, Effective Mass, Anticipation, Side-Cutting

\section{Introduction}

The Lower extremity injuries commonly occurs in soccer player, and female athletes such as soccer, basketball and team handball, have been demonstrated to be at a 2.3-9.7 times higher risk of anterior cruciate ligament(ACL) rupture compared with male athletes(Benjaminse, Gokeler, Fleisig, Sell \& Otten, 2011; Scott, Kelly, Cheryl, William \& Kevin, 2009). Non-contact ACL injuries in soccer players most often occurs while suddenly changing direction, fast decelerating or dynamic landing(Beaulieu, Lamontagne, $\mathrm{Xu}$, \& Scott et al., 2009). Also, cutting or landing maneuvers performed without adequate planning or unanticipated cutting may

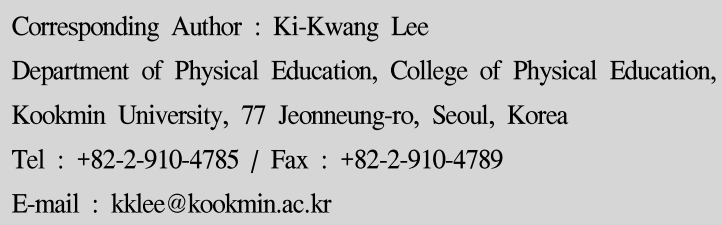

increase the risk of non-contact knee injury(Besier, Lloyd, Ackland \& Cochrane, 2001; Houck, Duncan, Haven, 2006; Borotikar, Newcomer, Koppes \& McLean, 2008; Beaulieu et al., 2009). One of the reasons is that probably due to the small amount of time to make appropriate postural adjustments before performance of the task, such as the position of the foot on the ground relative to the body center of mass(Besier et al., 2001).

Several studies has reported that neuromuscular response at early stance phase during cutting or landing task is very important, because the ACL is usually injured in early stance just after initial contact with the ground(Ford, Myer, Toms, Hewett, 2005; Scott et al., 2009). The impact forces that occur due to the collision of the foot with the ground in running shows usually two peaks, a first peak, the impact force peak and a second peak, the active force peak (Nigg \& Liu, 1999; Wakeling, Liphard \& Nigg, 2003). This distinction between impact force peaks was made because the time required for a muscle to react to the sensory signal generated during 
the impact(Nigg \& Liu, 1999). Also, the impact force from the ground during landing affects as a load on the joints, ligaments and tendons(Nigg, 1980). The load transferred from the ground to the foot landing task causes by such as the instantaneous load, overload, the lack of flexibility, and the impact load caused by the repeatedly behavior is associated with lower limb injury(Nigg, 1980).

The impact force and the shock wave are considered one of the primary etiological agents in degenerative joint diseases and injuries of the musculo-skeletal system(Chi \& Schmitt, 2005). Also, the impact force at early stance phase is very important to investigate the lower limb injury not only running but also other movements including cutting or landing. The side cutting maneuver is typically combined with running, and it exposes to high risk rate of the lower limb. A significant amount of research has been conducted in order to determine strategies for injury prevention; yet, the incidence remains high(Benjaminse et al., 2011). The impact force should be expected to calculate the effective mass that get the impact force by calculating of a part of body to know the amount of energy absorbed or delivered. Not much study has documented these values in living subjects during normal locomotion, because it is necessary to include the effective mass $\left(\mathrm{M}_{\mathrm{eff}}\right)$ in any equations calculating mechanical energy(Chi \& Schmitt, 2005). But with known foot landing velocity, $\mathrm{M}_{\text {eff }}$ can be calculated by equating work done and kinetic energy of the foot mass(Chi \& Schmitt, 2005).

Therefore, the aim of this study is to investigate the anticipatory effects on effective mass during side-cutting, and to examine the relationship between knee joint maximum angles and the effective mass.

\section{Methods}

\section{Subjects}

Thirteen right-leg dominant female elite college soccer players (age 19.2 \pm 0.4 years, height $162.5 \pm 5.2 \mathrm{~cm}$, weight $55.2 \pm 4.4 \mathrm{~kg}$, career $7.5 \pm 1.4$ years) were recruited for the this study. None of the subjects had suffered from any previous knee or any lower limb injuries Informed written consent was obtained from each participant.

\section{Experimental Setting}

Six cameras, $200 \mathrm{~Hz}$ Vicon motion analysis system(T-40, Vicon Motion System Limited, Oxford, UK) were synchronized with a force platform(2000Hz, AMTI BP-600900, Advanced Mechanical Technology Inc, Watertown, USA) to obtain kinetic and kinematic data. Vicon system cameras were set up around a third-generation artificial soccer surface(Campo, Allgreen, Korea) with a cryogenic rubber infill and an open polyethylene fiber matrix. The weight of the surface ensured stability. A minimum distance of $7 \mathrm{~m}$ was provided for each subject to approach the force platform and a minimum runoff distance of $5 \mathrm{~m}$.

Each subject wore the boots that they wear during daily training. One pair of photoelectric cell(BX5M-MDT, Autonics, Buchon, Korea) were placed at a width of 2.5 apart and placed $2 \mathrm{~m}$ in front of the force platform(Beaulieu et al., 2009). To create an unanticipated environment, the athletes triggered, $2 \mathrm{~m}$ before the force platform by means of a photoelectric cell an LED equipped with left and right light indicating which task to perform. When the left color appeared, the participants executed the side-cutting task; on a right color, they executed the cross-cutting task. The sequence of the color was random. A total of $16(14 \mathrm{~mm})$ retro-reflective markers were attached to the lower body of each subject using double-sided tape and elastic tape on their bases according to the Vicon "plug in gait" lower limb marker set.

\section{Data Collection}

The athletes were required to perform three successful trials for anticipated side cutting (AC) and unanticipated side cutting (UC) maneuvers. Specifically they had to approach the force platform at their maximum speed, step on the force platform with their right foot and change direction to the left $45^{\circ}$ (side-cut) from the line of direction of the approach run and for unanticipated contion, subjects performed left $45^{\circ}$ (side-cut) or right $45^{\circ}$ (cross-cut) angle with left and right light indicating signal. The subjects were permitted to perform the cutting movement with correct foot placement on the

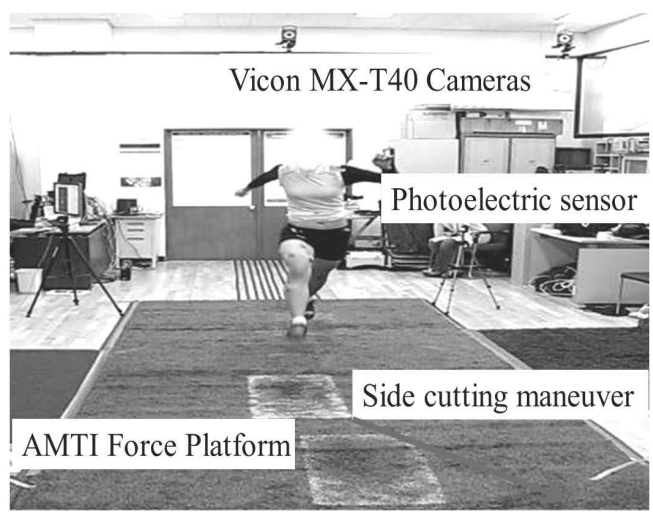

Figure 1. Experimnetal set up for side-cutting maneuver 
force platform based on their stride lengths and starting positions $<$ Figure 1>. To prevent fatigue, the subjects were required to rest at least 2 minutes between each trial

To analyze the knee angle and effective mass, the kinematics data were collected and the effective mass $\left(\mathrm{M}_{\mathrm{foot}}\right)$ were calculated, using the form(Liberman, Venkadesan, Werbel, Daoud, Andrea, Davis, Mang, Pitsiladis, 2010) following as

$$
M_{e f f}=\frac{\int_{0-}^{T} F_{Z}(t) d t}{-v_{\text {foot }}+g T}
$$

Where $\mathrm{Fz}(\mathrm{t})$ is the time-varying vertical ground reaction force, $0^{-}$is the instance of time of time before impact, $\mathrm{T}$ is duration of the impact transient, $v_{\text {foot }}$ is the vertical speed of the foot just before impact and $\mathrm{g}$ is the acceleration due to gravity at the Earth's surface(Lieberman et al., 2010). However, in this current study, the time-varying resultant ground reaction force $(\mathrm{FR}(\mathrm{t}))$ was used than the time-varying vertical force. Because, the experimental task of Lieberman et al.,(2010) testing conditions involved subjects running straight, but in this current study, various directions were included, so the $\mathrm{M}_{\text {eff }}$ could not explain only one direction force component. The effective mass $\left(\mathrm{M}_{\text {foot }}\right)$ for side-cutting movement can be calculated, using experimental data from equation, as

$$
M_{\text {foot }}=\frac{\int_{0-}^{T} F_{R}(t) d t}{-v_{\text {foot }}+g T}
$$

\section{Data Analysis}

To obtain the knee maximum angles and $\mathrm{M}_{\text {foot }}$, three-dimensional kinetic and kinematics data were recorded by the Vicon software The ground reaction force data were normalized according to body mass (\%BM). In the anticipated cutting, one successful trial data selected for analysis, and in the unanticipated cutting trials, the average of the two successful trials were used. The deceleration phase was defined as the time from foot contact to maximum flexion angle of the right leg was selected for the analysis of the $\mathrm{M}_{\text {foot }}$. Foot contact was defined as the instant when the vertical GRF rose above and below $10 \mathrm{~N}$ respectively(Borotikar et al., 2008).

To analyze the $\mathrm{AC}$ and $\mathrm{UC}$ condition, univariate repeated-measures analysis of variance(ANOVA) was used to compare maximun knee flexion /extension, valgus/varus and internal/external rotation angles and, $\mathrm{M}_{\mathrm{foot}}$. To identify the relationship between knee maximum angles and $\mathrm{M}_{\mathrm{foot}}$, the correlation analysis was implemented. The SASW 18.0 statistic analysis package software was used and, a significant level of $a<.05$. was established.

\section{Results}

\section{Knee Maximum Flexion Angles}

Peak knee flexion angles were significantly different between $\mathrm{AC}$ and UC ( $p=.002)$. There were higher peak knee flexion angles in UC condition compared with AC. However, mean knee flexion angles at initial contact and toe-off stages were not significantly different between $\mathrm{AC}$ and UC. Knee kinematic values for each stage of stance are shown in (Table 1).

Table 1. Maximum angles of the knee joint

\begin{tabular}{ccc}
\hline & \multicolumn{2}{c}{ Mean angles $\pm S D$ of the knee joint $\left(^{\circ}\right)$} \\
\cline { 2 - 3 } Maximum & $p$ \\
\hline AC & $56.91 \pm 7.68$ \\
UC & $62.91 \pm 6.19$ & .002 \\
\hline \multicolumn{3}{c}{ Varus(+) / Valgus(-) } \\
AC & $-7.33 \pm 6.84$ \\
UC & $-9.31 \pm 7.69$ \\
\hline \multicolumn{3}{c}{ Internal rotation(+) } \\
AC & $16.13 \pm 5.10$ \\
UC & $17.77 \pm 5.78$ \\
\hline
\end{tabular}

AC: Anticipated side-cutting, UC: Unanticipated side-cutting.

\section{Knee Maximum Varus/Valgus Angles}

The valgus angles increased when the task were performed in the UC condition compared with the $\mathrm{AC}(p=.042)$. The peak knee varus angles were no significant different between $\mathrm{AC}$ and UC. Also, there were no significant differences between the mean angles at initial contact and toe-off stages.

\section{Knee Maximum Internal/External Rotation Angles}

For the internal rotation angles at maximum and toe-off stages 
there were significant differences between $\mathrm{AC}$ and UC. For the peak internal rotation angles of the UC condition they showed increased mean angles( $p=.024)$, and the angles of the toe-off stage indicated significantly more external rotation in the $\mathrm{UC}$ than the $\mathrm{AC}$ ( $p=$.043).

\section{Effective mass}

Significantly greater $\mathrm{M}_{\mathrm{foot}}$ means occurred at the UC condition to compared with $\mathrm{AC}(p=.019)$. $\mathrm{M}_{\text {foot }}$ for each cutting condition is shown in (Table 2).

Table 2. Mean $\pm S D$ of the $\mathrm{M}_{\mathrm{fox}}$ during deceleration phase.

\begin{tabular}{cc}
\hline & Mean $\pm S D$ of the $\mathrm{M}_{\text {foot }}$ (\% Body Mass) \\
\hline \hline $\mathrm{AC}$ & $1.22 \pm 0.20$ \\
$\mathrm{UC}$ & $1.48 \pm 0.40$ \\
$p$ & .019 \\
\hline AC: Anticipated side-cutting, UC: Unanticipated side-cutting. \\
$\mathrm{M}_{\text {foot: }}$ effective mass.
\end{tabular}

\section{Correlations between knee maximum angles and $\mathbf{M}_{\text {foot }}$}

The peak knee flexion angles correlated with effective mass for the $\mathrm{AC}$ condition ( $p=.014)$, and there was no correlation in UC condition $(p=.824)<$ Table $3>$. Knee maximum varus, valgus and internal rotation angles had no significant correlation within $\mathrm{AC}$ and $\mathrm{UC}$ conditions $<$ Figure 2>. Indicates an increased knee peak angle following higher $\mathrm{M}_{\text {toot }}$.

Table 3. Correlation between knee joint angle and effective foot mass during deceleration phase.

\begin{tabular}{cccc}
\hline condition & \multicolumn{2}{c}{ knee joint angle } \\
\hline \hline & & pearson $\mathrm{r}$ & $p$ \\
$\mathrm{AC}$ & $\mathrm{M}_{\text {foot }}$ & .661 & 014 \\
$\mathrm{UC}$ & & .069 & .824 \\
\hline
\end{tabular}

AC: Anticipated side-cutting, UC: Unanticipated side-cutting. $\mathrm{M}_{\text {foot: }}$ effective mass

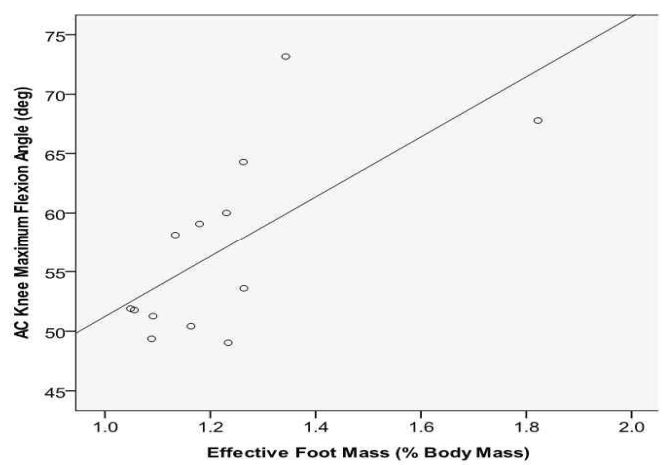

Figure 2. The graph of correlations between knee maximum flexion angle and $\mathrm{M}_{\mathrm{eff}}$ (effective mass)

\section{Discussion}

Our goal was to estimate the amount of the $\mathrm{M}_{\mathrm{foot}}$ depending on the anticipation or not, and to examine the co-relationship between the amount of foot mechanical energy and knee joint angle for shock absorb during decelerate landing phase of side-cutting.

The results of this study suggest that it is appropriate to use different $\mathrm{M}_{\text {foot }}$ for different anticipation conditions: about $1.22 \%$ $\mathrm{BM}$ for $\mathrm{AC}, 1.48 \% \mathrm{BM}$ for UC $(p=.019)$. There were significant correlations only between knee joint maximum flexion angles and the magnitude of $\mathrm{M}_{\mathrm{foot}}$ in $\mathrm{AC}$ ( $\left.p=.013\right)$, no statistical differences in maximum valgus/varus and rotation angles. In UC condition, there were no significant correlations between knee joint maximum flexion, valgus/varus and rotation angles and the $\mathrm{M}_{\mathrm{foot}}$.

The maximum knee joint flexion angles in $\mathrm{AC}$ increased with an increase in magnitude of higher $\mathrm{M}_{\mathrm{foot}}$. That means subjects can expect the amount of impact before landing, and they can control the shock absorption by flex their knee joint in anticipation. However, no positive correlations between $\mathrm{M}_{\mathrm{foot}}$ and maximum knee flexion angle in UC means that shock absorb during landing in UC can not be controlled by knee flexion, and the unexpected load affect was more irregular on the knee joint.

The impact force during landing was closely related to the risk of lower limb injury. Ground reaction forces typically have an impact peak after heel-strike due to the collision of the foot with the ground(Wakeling et al., 2003). The impact force occurs within $50 \mathrm{~ms}$ after first contact and causes shock waves to travel through both the soft-tissues and skeletal components of the body (Wakeling et al., 2003).

Lieberrman et al.,(2010) studied about effective mass during landing and they reported barefoot runners who fore-foot strike generate smaller collision forces than shod rear-foot strikers, that means a more plantar flexed foot at landing and more the ankle compliant during impact, decreasing the effective mass of the body that collides with the ground, They also demonstrated that the effective mass acting on the foot is proportional to the impact force and loading rate. As the loading rate is related with time that means the landing with a high loading rate leaves less reaction time for shock absorption. In other words, it is same as not enough control time to reduce the higher effective mass

Chi et al.,(2004). collected ground reaction forces, pad deformation, lower limb kinematics and the effect of knee angles on effective foot mass $\left(\mathrm{M}_{\mathrm{eff}}\right)$ during impact phase of barefoot walking, running, and 
crouched walking. The magnitude of $\mathrm{M}_{\text {eff }}$ as a percentage of body mass (BM) varied with knee angle at impact and significantly differs among gaits: $6.3 \% \mathrm{BM}$ in walking, 5.3\% $\mathrm{MB}$ in running, and $3.7 \% \mathrm{BM}$ in walking, 5.3 \% $\mathrm{BM}$ in running, and $3.7 \% \mathrm{BM}$ in crouched walking (Chi and Schmitt, 2005). These results provide insights that position might affect $\mathrm{M}_{\text {eff }}$ during walking.

The result of this study suggest that $\mathrm{M}_{\text {foot }}$ showed higher effective mass of foot in UC than the AC, and knee joint load can be explained by the $\mathrm{M}_{\text {foot }}$ in AC. However in UC condition, it was difficult to explain the knee joint load by $\mathrm{M}_{\text {foot }}$ because there were no significant co-relationship between $\mathrm{M}_{\mathrm{foot}}$ and knee maximum angles.

Future research should focus on the relationship of $\mathrm{M}_{\mathrm{fot}}$ and knee angles especially, how changes in posture moderate impact transient in unanticipated condition.

\section{Conclusion}

Peak knee flexion angles were significantly different between $\mathrm{AC}$ and $\mathrm{UC}$ condition $(p<.05)$. The knee maximum valgus angles increased when the cuttings task were performed in the UC conditions compared with the AC. The internal knee rotation angles at maximum and toe-off events had significant differences between $\mathrm{AC}$ and UC. The effective mass $\left(\mathrm{M}_{\mathrm{foot}}\right)$ during UC was greater than during $\mathrm{AC}$. There was a relationship between effective mass and knee flexion angle in AC. In the unanticipated cutting movements, knee movement might be able to change for shock absorption strategy.

\section{References}

Beaulieu, M. Lamontagne, M., \& Xu, L.(2009). Lower limb muscle activity and kinematics of an unanticipated cutting manoeuvre: a gender comparison. Knee Surgery, Sports Traumatology, Arthroscopy, 17, 968-976.

Benjaminse, A., Gokeler, A., Fleisig, G., Sell, T., \& Otten, B. (2011). What is the true evidence for gender-related differences during plant and cut maneuvers? A systematic review. Knee Surgery, Sports Traumatology, Arthroscopy, 19, 42-54.

Besier, T. F., Lloyd, D. G., Ackland, T. R., \& Cochrane, J. L.
(2001). Anticipatory effects on knee joint loading during running and cutting maneuvers. Medicine and Science in Sports and Exercise, 33, 1176-1181.

Borotikar, B. S., Newcomer, R., Koppes, R., \& McLean, S. G. (2008). Combined effects of fatigue and decision making on female lower limb landing postures: Central and peripheral contributions to ACL injury risk. Clinical Biomechanics, 23, 81-92.

Chi, K. J., \& Schmitt, D.(2005). Mechanical energy and effective mass during impact loading of walking and running. Journal of Biomechanics, 38, 1387-1395.

Ford, K. R., Myer, G. D., Toms, H. E., \& Hewett, T. E.(2005). Gender Differences in the Kinematics of Unanticipated Cutting in Young Athletes. Medicine and Science in Sports and Exercise, 37, 124-129.

Houck, J. R., Duncan, A., \& Haven, K. E. D.(2006). Comparison of frontal plane trunk kinematics and hip and knee moments during anticipated and unanticipated walking and side step cutting tasks. Gait \& Posture, 24, 314-322.

Lieberman, D. E., Venkadesan, M., Werbel, W. A., Daoud, A. I., D/'Andrea, S., Davis, I. S., et al. (2010). Foot strike patterns and collision forces in habitually barefoot versus shod runners. Nature, 463, 531-535.

Nigg, B. M., and Liu, W.(1999). The effect of muscle stiffness and damping on simulated impact force peaks during running. Journal of Biomechanics, 32, 849-856.

Nigg, D. B. M.(1980). Quantifying load on the human body. Journal of Biomechanics, 13, 636-636.

Scott, C. L., Kelly, A. M., Cheryl, L. H. K., William, D. S., \& Kevin, J. D.(2009). Gender differences exist in neuromuscular control patterns during the pre-contact and early stance phase of an unanticipated side-cut and cross-cut maneuver in 15-18 years old adolescent soccer players. Journal of Electromyography and Kinesiology, 19(e), 370-379.

Wakeling, J. M., Liphardt, A. M., \& Nigg, B. M.(2003). Muscle activity reduces soft-tissue resonance at heel-strike during walking. Journal of Biomechanics, 36, 1761-1769. 